\title{
SPLITTING UNIVERSAL BUNDLES OVER FLAG MANIFOLDS
}

\author{
R. E. STONG
}

\begin{abstract}
Let $\mathbf{F}$ be one of the fields $\mathbf{R}, \mathbf{C}$, or $\mathbf{H}$ and correspondingly let $\mathbf{F} G$ be $O, U$, or $\mathrm{Sp}$, i.e. the orthogonal, unitary, or symplectic group. Over the flag manifold $\mathbf{F} G\left(n_{1}+\cdots+n_{k}\right) / \mathbf{F} G\left(n_{1}\right) \times \cdots \times \mathbf{F} G\left(n_{k}\right)$ one has vector bundles $\gamma_{i}$ over $F$ of dimension $n_{i}, 1 \leqslant i \leqslant k$. This paper determines all cases in which $\gamma_{i}$ decomposes nontrivially as a Whitney sum.
\end{abstract}

1. Introduction. Let $\mathbf{F}$ be one of the fields $\mathbf{R}, \mathbf{C}$, or $\mathbf{H}$, and correspondingly let $\mathbf{F} G$ be $O, U$, or $\mathrm{Sp}$, i.e. the orthogonal, unitary, or symplectic group. If $n=n_{1}+n_{2}$ $+\cdots+n_{k}, 1 \leqslant n_{i}$, let

$$
\mathbf{F M}\left(n_{1}, n_{2}, \ldots, n_{k}\right)=\mathbf{F} G(n) / \mathbf{F} G\left(n_{1}\right) \times \mathbf{F} G\left(n_{2}\right) \times \cdots \times \mathbf{F} G\left(n_{k}\right)
$$

be the manifold of flags, consisting of $k$ mutually orthogonal F-subspaces of $\mathbf{F}^{n}$ with the $i$ th subspace having dimension $n_{i}$. Over $\mathbf{F M}\left(n_{1}, n_{2}, \ldots, n_{k}\right)$, one has vector bundles $\gamma_{i}, 1 \leqslant i \leqslant k$, over $\mathbf{F}$ with the fiber dimension of $\gamma_{i}$ being $n_{i}$, with the total space consisting of pairs $(V, x)$, where $V=\left(V_{1}^{n_{1}}, V_{2}^{n_{2}}, \ldots, V_{k}^{n_{k}}\right)$ is a flag and $x \in V_{i}^{n_{i}}$ is in the $i$ th subspace.

The purpose of this note is to determine all cases in which it is possible to have decompositions of the bundles $\gamma_{i}$ into nontrivial Whitney sums of F-subbundles. Equivalently, let $n_{i}=n_{1}^{i}+n_{2}^{i}+\cdots+n_{j_{i}}^{i}, 1 \leqslant n_{j}^{i}, 1 \leqslant j_{i}$, be partitions of the $n_{i}$. One then has a fibering

$$
\pi: \mathbf{F M}\left(n_{1}^{1}, \ldots, n_{j_{1}}^{1}, \ldots, n_{1}^{k}, \ldots, n_{j_{k}}^{k}\right) \rightarrow \mathbf{F M}\left(n_{1}, n_{2}, \ldots, n_{k}\right),
$$

and this note will determine all cases in which $\pi$ has a section.

This problem is suggested by work of Glover and Homer [1, 2], and generalizes the solution for projective spaces (the case $F M\left(1, n_{2}\right)$ ) in [3] and for Grassmannians (the case $\mathbf{F M}(p, q), 2 \leqslant p \leqslant q)$ in [5]. In essence, this note reduces the general problem to those special cases, or more precisely to the solutions given for those cases. The easiest case is $\mathbf{F}=\mathbf{H}$, which is in fact completely trivial, while the case $\mathbf{F}=\mathbf{R}$ is the most difficult and requires a few new thoughts. The paper will be organized by taking these cases in the order of increasing difficulty.

In the final section, it will be shown that there are no real splittings of the universal bundles over quaternionic Grassmannians $\mathbf{H} M(p, q), 2 \leqslant p \leqslant q$. This case was not covered in [5], and serves to complete those results.

The author is indebted to the National Science Foundation for financial support during this work.

Received by the editors May 4, 1981 and, in revised form, June 23, 1981.

1980 Mathematics Subject Classification. Primary 55R40.

(C)1982 American Mathematical Society $0002-9939 / 81 / 0000-0354 / \$ 02.25$ 
2. Easy results: the case $F=H$.

LEMMA 1. Let $1 \leqslant n_{i}, 1 \leqslant i \leqslant k$, with $2 \leqslant k$. Let $n_{i}=n_{1}^{i}+n_{2}^{i}+\cdots+n_{j_{i}}^{i}$, with $1 \leqslant n_{j}^{i}, 1 \leqslant j_{i}$, and suppose that $j_{k}>1$; i.e. one has a genuine partition of $n_{k}$. If the fibering

$$
\pi: \mathbf{F} M\left(n_{1}^{1}, \ldots, n_{j_{1}}^{1}, \ldots, n_{1}^{k}, \ldots, n_{j_{k}}^{k}\right) \rightarrow \mathbf{F} M\left(n_{1}, n_{2}, \ldots, n_{k}\right)
$$

has a section, then the fibering

$$
\pi^{\prime}: \mathbf{F} M(1 \underbrace{1, \ldots, 1}_{r}, n_{1}^{k}, \ldots, n_{j_{k}}^{k}) \rightarrow \mathbf{F M}(\underbrace{1, \ldots, 1}_{r}, n_{k})
$$

also has a section, provided $r \leqslant n_{1}+n_{2}+\cdots+n_{k-1}$.

Proof. If $\pi$ has a section, $\gamma_{k}$ splits over $\operatorname{FM}\left(n_{1}, \ldots, n_{k}\right)$ and hence the pullback over $\operatorname{FM}\left(1, \ldots, 1, n_{k}\right)$ ( $r$ ones) admits the same decomposition.

Proposition 1. In the quaternionic case $(\mathbf{F}=\mathbf{H})$, none of the genuine fiberings $\pi$ has a section.

Proof. If $\pi: \mathbf{H} M\left(n_{1}^{1}, \ldots, n_{j_{k}}^{k}\right) \rightarrow \mathbf{H} M\left(n_{1}, \ldots, n_{k}\right)$, with $2 \leqslant k, j_{k}>1$, has a section, then by the lemma, so does

$$
\pi^{\prime}: \mathbf{H} M\left(1, n_{1}^{k}, \ldots, n_{j_{k}}^{k}\right) \rightarrow \mathbf{H} M\left(1, n_{k}\right)=\mathbf{H} P^{n_{k}} .
$$

However, the bundle $\gamma_{n}$ over $\mathbf{H} \boldsymbol{P}^{n}$ never admits a proper Whitney sum decomposition over $\mathbf{H}$ (or even over $\mathbf{C}$ ) by [3].

3. Semisimple results: the case $\mathbf{F}=\mathbf{C}$.

LEMMA 2. The fibering

$$
\pi: \mathbf{C M}\left(1,1, m_{1}, \ldots, m_{j}\right) \rightarrow \mathbf{C M}(1,1, m)
$$

with $j>1$ has no section.

Proof. Suppose there was a section. Over $\mathbf{C M}(1,1, m)$, one has two line bundles $\gamma_{1}$ and $\gamma_{2}$ and an $m$-plane bundle $\gamma_{3}$, with the bundle $\gamma_{3}$ having a proper Whitney sum decomposition $\gamma_{3}=\xi^{p} \oplus \eta^{q}, p+q=m, p \leqslant q$. By Lemma 1 , the same decomposition exists over $C M(1, m)$. Applying the results of [3], $m$ is odd and $p=1$, $q=m-1$. Further, over $\mathbf{C} M(1, m)$, the line bundle $\xi$ is the complex conjugate of the line bundle $\gamma_{1}$.

Thus, over $\mathbf{C} M(1,1,2 s+1)$, the only possible decomposition is $\gamma_{3} \cong \xi^{1} \oplus \eta^{2 s}$. Let $c_{1}\left(\gamma_{1}\right)=x$ and $c_{1}\left(\gamma_{2}\right)=y$. Using the two different inclusions of $\mathbf{C} M(1,2 s+1)$ in C $M(1,1,2 s+1)$, it is immediate that $c_{1}(\xi)=-(x+y)$. Now examining the arguments in [5], the argument that $\gamma^{p}$ over $\mathbf{C} M(2, p)$ cannot split was actually done by calculation in $\mathbf{C P}\left(\gamma^{2}\right)=\mathbf{C} M(1,1, p)$. Using Stiefel-Whitney and Pontrjagin class calculations, it was shown that the pullback of $\gamma^{p}$ to $\mathrm{CP}\left(\gamma^{2}\right)$ could not contain a line bundle with Chern class $-(x+y)$. 
Proposition 2. In the complex case $(\mathbf{F}=\mathbf{C})$, the only genuine fiberings $\pi$ having sections are the fiberings

$$
\pi: \mathbf{C M}(1,1,2 s) \rightarrow \mathbf{C M}(1,2 s+1) .
$$

Proof. If $\pi: \mathbf{C} M\left(n_{1}^{1}, \ldots, n_{j_{k}}^{k}\right) \rightarrow \mathbf{C M}\left(n_{1}, \ldots, n_{k}\right)$ has a section, then Lemma 1 gives a section of $\pi^{\prime}$ for $r \leqslant n_{1}+\cdots+n_{k-1}$. By Lemma 2, $r$ must be less than 2, and the only splittings occur in the complex projective space case.

4. Hard results: the case $F=R$.

LEMMA 3. If the fibering

$$
\pi: \mathbf{R} M\left(1,1, m_{1}, \ldots, m_{j}\right) \rightarrow \mathbf{R} M(1,1, m)
$$

with $j>1$ has a section, then $\left(m_{1}, m_{2}, \ldots, m_{j}\right)=(1, m-1)$ and, in fact, $m=5$.

Proof. Suppose there is a section, so that $\gamma_{3}$ over $\mathbf{R} M(1,1, m)$ has a proper Whitney sum decomposition $\gamma_{3}=\xi^{p} \oplus \eta^{q}, p+q=m, p \leqslant q$. Let $w_{1}\left(\gamma_{1}\right)=x, w_{1}\left(\gamma_{2}\right)$ $=y$ be the first Stiefel-Whitney classes of the two line bundles. Then $H^{*}\left(\mathbf{R} M(1,1, m) ; Z_{2}\right)$ is the $Z_{2}$ polynomial ring on $x$ and $y$ modulo relations in dimensions $m+1$ and higher.

Claim. There are integers $a, b, c$ with $w(\xi)=(1+x)^{a}(1+y)^{b}(1+x+y)^{c}$. To see this, one has maps

$$
\begin{aligned}
& \mathbf{R} P^{\infty} \times \mathbf{R} P^{\infty} \\
& \underset{f \uparrow}{ } \\
& \mathbf{R} M(1,1, m) \stackrel{g}{\rightarrow} \quad B O_{p}
\end{aligned}
$$

where $f$ classifies $\gamma_{1}$ and $\gamma_{2}$ and $g$ classifies $\xi^{p}$. In cohomology one has

$$
\begin{aligned}
& H^{*}\left(\mathbf{R} P^{\infty} \times \mathbf{R} P^{\infty} ; Z_{2}\right) \\
& f^{*} \downarrow \\
& H^{*}\left(\mathbf{R} M(1,1, m) ; Z_{2}\right) \stackrel{g^{*}}{\leftarrow} H^{*}\left(B O_{p} ; Z_{2}\right)
\end{aligned}
$$

with $f^{*}$ an isomorphism through dimension $m \geqslant 2 p$. Now $H^{*}\left(B O_{p} ; Z_{2}\right)$ is generated by $w_{1}, w_{2}, \ldots, w_{p}$ with relations given by the $\mathrm{Wu}$ formulae for $\mathrm{Sq}^{i} w_{j}(1 \leqslant i \leqslant j)$, which occur in dimensions at most $2 p$, and so one has a lifting

$$
\begin{array}{ccc}
H^{*}\left(\mathbf{R} P^{\infty} \times \mathbf{R} P^{\infty} ; Z_{2}\right) & \\
f^{*} \downarrow & \nwarrow \phi \\
H^{*}\left(\mathbf{R} M(1,1, m) ; Z_{2}\right) & \stackrel{g^{*}}{\leftarrow} & H^{*}\left(B O_{p} ; Z_{2}\right)
\end{array}
$$

with $\phi$ being a homomorphism of algebras over the Steenrod algebra. Now Patterson [4] shows that any such homomorphism

$$
\tilde{\phi}: H^{*}\left(B O ; Z_{2}\right) \rightarrow H^{*}\left(\mathbf{R} P^{\infty} \times \mathbf{R} P^{\infty} ; Z_{2}\right)
$$

has the form $\tilde{\phi}(w)=(1+x)^{a}(1+y)^{b}(1+x+y)^{c}$, which gives the claim. 
Claim. $w(\xi)$ is symmetric.

To see this, let $f_{1}, f_{2}: \mathbf{R} M(1, m) \rightarrow \mathbf{R} M(1,1, m)$ be the two inclusions, for which $f_{1}^{*}\left(\gamma_{1}\right) \cong \gamma^{1}, f_{1}^{*}\left(\gamma_{2}\right) \cong 1=$ trivial and $f_{2}^{*}\left(\gamma_{1}\right)=1, f_{2}^{*}\left(\gamma_{2}\right)=\gamma^{1}$, and hence $f_{i}^{*}\left(\gamma_{3}\right)=\gamma^{m}$ $\supset f_{i}^{*}\left(\xi^{p}\right)$. Let $z=w_{1}\left(\gamma^{1}\right)$, one has from [3] that $w\left(f_{i}^{*}\left(\xi^{p}\right)\right)=(1+z)^{p}$. Thus $(1+z)^{a}=(1+z)^{p} /(1+z)^{c}=(1+z)^{b}$ in $H^{*}\left(\mathbf{R} M(1, m) ; Z_{2}\right)$, so that the coefficient of $x^{t}$ in $(1+x)^{a}$ is the same as that of $y^{t}$ in $(1+y)^{b}$ for $t \leqslant m$. Thus interchanging $x$ and $y$ does not change $w_{1}(\xi), \ldots, w_{p}(\xi)$, and $w(\xi)$ is symmetric in $x$ and $y$.

Thus $w\left(\xi^{p}\right)$ is symmetric in $x$ and $y$, and hence comes from $H^{*}\left(\mathbf{R} M(2, m) ; Z_{2}\right)$. The arguments in [5] about splitting $\gamma^{m}$ over $\mathbf{R} M(2, m)$ then apply to give $m=5$ and $p=1$ and also $w(\xi)=1+(x+y)$. Notice that Lemma 3 of [5] is purely algebraic by the note after the proof and that the geometric part of Lemma 4 about sectioning $O(n+2) / O(n-1) \rightarrow O(n+2) / O(n)$ occurs over the space of flags.

LEMMA 4. If the fibering

$$
\pi: \mathbf{R} M(1 \underbrace{\ldots, \ldots, 1}_{r}, m_{1}, \ldots, m_{j}) \rightarrow \mathbf{R} M(1 \underbrace{1, \ldots, 1}_{r}, m)
$$

with $j>1$ has a section, then $r \leqslant 3$.

Proof. By Lemma 3, $m=5,\left(m_{1}, \ldots, m_{j}\right)=(1,4)$, using Lemma 1 implicitly. Further, with Lemma 1 , one may reduce the case $r \geqslant 4$ to $r=4$. However, the argument for splitting $\gamma^{5}$ over $\mathbf{R} M(4,5)$ in [5] shows that no decomposition can exist.

PROPOSITION 3. In the real case $(\mathbf{F}=\mathbf{R})$, the only genuine fiberings

$$
\pi: \mathbf{R} M\left(n_{1}^{1}, \ldots, n_{j_{k}}^{k}\right) \rightarrow \mathbf{R} M\left(n_{1}, \ldots, n_{k}\right)
$$

$\left(j_{k}>1\right)$ which have sections are:

$$
\text { (a) } \pi: \mathbf{R} M\left(1, n_{1}^{k}, \ldots, n_{j_{k}}^{k}\right) \rightarrow \mathbf{R} M\left(1, n_{k}\right),
$$

which occur for $n_{k}$ odd and $\sup \left(n_{j}^{k}\right) \geqslant\left(n_{k}+1\right)-\rho\left(n_{k}+1\right)$, and

$$
\text { (b) } \pi: \mathbf{R} M\left(n_{1}, \ldots, n_{k-1}, 1,4\right) \rightarrow \mathbf{R} M\left(n_{1}, \ldots, n_{k-1}, 5\right)
$$

with $n_{1}+\cdots+n_{k-1} \leqslant 3$.

Proof. By Lemmas 1 and $4, n_{1}+\cdots+n_{k-1}=r \leqslant 3$. The possible splittings for $r=1$ were given in [3]. For $r \geqslant 2$, Lemma 3 gives $n_{k}=5,\left(n_{1}^{k}, \ldots, n_{j_{k}}^{k}\right)=(1,4)$. Pulling back the splitting of $\gamma^{5}$ over $\mathbf{R} M(3,5)$, given in [5], gives all splittings of (b). It is clear that none of the bundles $\gamma_{1}, \ldots, \gamma_{k-1}$ over $\mathbf{R} M\left(n_{1}, \ldots, n_{k-1}, 5\right)$ with $n_{1}+\cdots+n_{k-1} \leqslant 3$ can decompose.

5. Real splittings for quaternionic Grassmannians. The purpose of this section is to complete the results of [5] by proving:

Proposition 4. Neither of the bundles $\gamma_{k}$ and $\gamma_{n}$ over $G_{k}\left(\mathbf{H}^{n+k}\right)=\mathbf{H} M(k, n)$ with $2 \leqslant k \leqslant n$ contains a proper real subbundle. 
Note. During the preparation of [5], I did not consider this case since it would easily follow if you could prove nonsplitting over $\mathbf{R}$ for $\gamma_{n}$ over $\mathbf{H} P^{n}$. Unfortunately, that is difficult, if indeed it is true. Thus, it seems worthwhile to do the Grassmannian case.

Proof. One has $H^{*}\left(G_{k}\left(\mathbf{H}^{n+k}\right) ; Z\right)=Z\left[p_{1}, \ldots, p_{k}, \bar{p}_{1}, \ldots, \bar{p}_{n}\right] /\{(p \bar{p})=1\}$ where $p=1+p_{1}+\cdots+p_{k}=\mathscr{P}^{s}\left(\gamma_{k}\right)$ and $1+\bar{p}_{1}+\cdots+\bar{p}_{n}=\mathscr{\rho} s\left(\gamma_{n}\right)$ are the symplectic Pontrjagin classes, so that

$$
c\left(\gamma_{k}\right)=1-p_{1}+p_{2}+\cdots+(-1)^{k} p_{k} .
$$

Since the Euler class $X\left(\gamma_{k}\right)=c_{2 k}\left(\gamma_{k}\right)=(-1)^{k} p_{k}$ is indecomposable, $\gamma_{k}$ admits no splitting.

If $\gamma_{n}=\xi^{j} \oplus \eta^{4 n-j}$ over $G_{k}\left(\mathbf{H}^{n+k}\right)$, then $\gamma_{n}=\xi^{j} \oplus \eta^{4 n-j}$ over $G_{2}\left(\mathbf{H}^{n+2}\right)$. Applying the mod 2 cohomology analysis [5] as for $G_{2}\left(\mathbf{R}^{n+2}\right)$ but with degrees multiplied by 4 , one has $n=2^{s}-3$ for some $(s \geqslant 3)$ and $j=4$. By properly choosing orientations

$$
\begin{aligned}
X\left(\xi^{4}\right) \cdot X\left(\eta^{4 n-4}\right) & =X\left(\gamma_{n}\right)=c_{2 n}\left(\gamma_{n}\right)=(-1)^{n} \bar{p}_{n} \\
& = \pm p_{1}^{n}+\text { terms divisible by } p_{2}
\end{aligned}
$$

and so $X\left(\xi^{4}\right)= \pm p_{1}$ is not divisible by any integer.

Applying the mod 3 reduced power operation,

$$
\mathscr{P}^{1}\left(p_{1}\right)=\mathscr{P}^{1}\left(x^{2}+y^{2}\right)=2 x^{4}+2 y^{4}=2\left(\left(x^{2}+y^{2}\right)^{2}-2 x^{2} y^{2}\right)=2 p_{1}^{2}-p_{2}
$$

(where $p=\left(1+x^{2}\right)\left(1+y^{2}\right)$ via the splitting principle). Since this is not a multiple of $p_{1}$, no vector bundle can have Euler class equal to $\pm p_{1}$.

\section{REFERENCES}

1. H. H. Glover and W. D. Homer, Self-maps of flag manifolds, Trans. Amer. Math. Soc. 267 (1981), 423-434.

2.

3. H. H. Glover, W. D. Homer and R. E. Stong, Splitting the tangent bundle of projective space, Indiana Univ. Math. J. (to appear).

4. R. R. Patterson, The square preserving algebra endomorphisms of $H^{*}\left(B O, Z_{2}\right)$, Quart. J. Math. 29 (1978), 225-240.

5. R. E. Stong, Splitting the universal bundles over Grassmannians, Algebraic Topology-Manifolds and Cell Complexes (G. M. Rassias, editor), North-Holland, Amsterdam (to appear).

Department of Mathematics, University of Virginia, Charlottesville, Virginia 22903 